\title{
Linear Bearing Fault Detection in Operational Condition Using Artificial Neural Network
}

\author{
Siwanu Lawbootsa ${ }^{1}$, Prathan Chommaungpuck ${ }^{1,2}$ and Jiraphon Srisertpol ${ }^{1 *}$
}

\author{
${ }^{1}$ School of Mechanical Engineering, Institute of Engineering, Suranaree University of Technology, Nakhon Ratchasima 30000, Thailand \\ ${ }^{2}$ Western Digital (Thailand) Co.ltd, Bang Pa-in Industrial Estate, Ayutthaya 13160, Thailand
}

\begin{abstract}
Nowadays, Factors of a competition of Hard Disk Drive (HDD) industry have reduced the cost of manufacturing process via increasing the rate of productivity and reliability of the automation machine. This paper aims to increase the efficacy of Condition-Based Maintenance (CBM) of linear bearing in Auto Core Adhesion Mounting machine (ACAM). The linear bearing faults considered in three causes such as healthy bearing, one ball bearing damage and one ball bearing damage with starved lubricant. The Fast Fourier Transform spectrum (FFT spectrum) can be detected for linear bearing faults and Artificial Neural Network (ANN) method used to analyze the cause of linear bearing faults in operational condition. The experimental results show the potential application of ANN and FFT spectrum technique as Fault Detection and Isolation (FDI) tool for linear bearing fault detection performance. The accuracy and decision making of ANN is enough to develop the diagnostic method for automation machine in operational condition.
\end{abstract}

\section{Introduction}

Hard Disk Drive (HDD) industry has a competition of product price and speed of reading and writing technology. The cost of manufacturing process and reliability of the automation machine are the important role to profit margin of a product. Head Gimbal Assembly (HGA) is the major component in HDD. HGA assembly process is used Auto Core Adhesion Mounting machine (ACAM) to attach the slider to suspension as shown in Fig.1, then connect the circuit between slider and suspension. ACAM machine is used to adhesive dispense and attach the slider to suspension with requiring both short cycle time and high accuracy. ACAM machine used the motor to transfer HGA clamping unit from position to position and linear bearing are integrated to support and friction reduction propose while movement. According, to the machine is continues run to support production. Linear bearing faults are the direct impact to ACAM machine efficacy reduction. The preliminary damage of the linear bearing must be detected before the machine breaks down. Thus, the Condition-Based Maintenance (CBM) of the linear bearing condition is necessary.

In order to detect faults of Rolling Element Bearings (REBs), faulty diagnoses are used. For automatic condition, monitoring provide by using Empirical Mode Decomposition (EMD) to extract the vibration signal. Artificial Neural Network (ANN) was applied to classify bearing defects. The result indicates that the classifier is promising for high accuracy of fault bearing detection [1].Fault diagnostics of ball bearing Adaptive Neuro Fuzzy Inference Systems (ANFIS) is used for automatically identify and classify of bearing fault it was found the accurate classification of $99.83 \%$ [2].

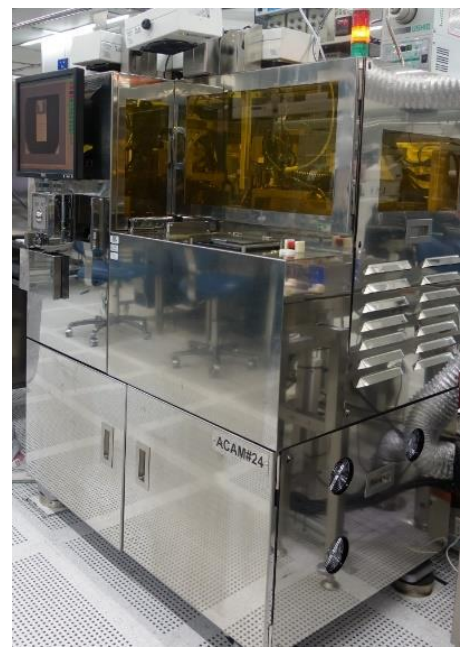

Fig.1 Auto Core Adhesion Mounting machine (ACAM)

The method presents the Artificial Neural Network (ANN) in machine condition monitoring and the result compares of simulation and experiment [3]. The paper on fault diagnosis of rolling bearings with recurrent neural

"Corresponding author: jiraphon@sut.ac.th 
network based autoencoders descript about the effect of ambient noise to extract the diagnostic result, therefore multiple sensors were used. Recurrent Neural Network (RNN) is used to a rotary machine for classifying fault. The model for predicting using Gated Recurrent Unit (GRU) based on this advantage is to utilize information from multiple sensors [4]. Zhiqiang Chen provided the deep neural network to rolling bearing fault diagnosis. Vibration signal of seven fault patterns in term of time and frequency domain was used for data training in three deep learning model which are consist of Stacked AutoEncoders (SAE), Deep Boltzmann Machines (DBM) and Deep Belief Networks (DBN) all models are efficient to classify. The accuracy achieved more than 99\% [5]. The detection of rolling bearing fault are used Continuous Deep Belief Network(CDBN) and Continuous Restricted Boltzmann machines(CRBMs) .The $\mathrm{CDBN}$ are optimized with genetic algorithm(GA)[6].The four condition of detection and classification in roller bearing: healthy, inner race defect, outer race defect and double holes in outer race used an Artificial Neural Network (ANN)[7].Fault diagnosis of rolling bearing are analysis by Hilbert Transform(HT) and Fast Fourier Transform(FFT).Artificial Neural Network used genetic algorithm into optimization[8]. In addition, the model of linear bearing was employed to detect fault form vibration signal. Statistical analysis is an extension method to analyze vibration moreover in order to detect the fault of linear bearing the vibration model has important [9]. Convolution neural network was provided due to fault classification of the rolling bearing by using vibration signal data. The training structure defines the hierarchical and back propagation process to classify the result indicate that the training simple affect to accuracy which is more sample the accuracy can be achieved [10]. Fault identification in the paper on sparse classification based on dictionary learning for planet bearing fault identification. Four conditions (sun gear, planet gear, ring gear and rolling gear) of fault was investigated. Chuan Zhao discusses spares method based on dictionary learning it has a merit of tackling raw inputs. On the other hand the vibration signal it cannot be learning to optimize inasmuch, map to transform the signal vector to a matrix form which retains the intrinsic fault feature information is used [11]. To analyze nonlinear vibration signal of rotating electrical machine three intelligence approach of the artificial neural network, genetic algorithms and active-set methods were proposed. The comparison is the numerical method with the help of different measures [12]. The Singular Spectral Analysis (SSA) is a time domain analysis technique this show the complex feature extraction. Roller element of bearing fault was detected by the applied neural network on singular spectral and evaluation using two experimental datasets. The result revealed that bearing fault diagnosis is simple, noise tolerant and efficient [13].

This paper aims to increase the efficacy of Condition-Based Maintenance (CBM) of linear bearing in Auto Core Adhesion Mounting machine (ACAM) by Fault Detection and Isolation (FDI) technique. The mechanical failure of linear bearing considered in three causes such as healthy bearing, one ball bearing damage and one ball bearing damage with starved lubricant. The analytical results of linear bearing fault by using the Fast Fourier Transform (FFT) spectrum and the Artificial Neural Network (ANN) methodology are proposed in this research.

\section{Theoretical backgrounds}

\subsection{Fault Detection and Isolation (FDI)}

Fault Detection and Isolation (FDI) technique, is widely used in many applications in control system. FDI is used to detect fault or failure then find the location or type of fault. So that feedback information to reconfiguration controller for eliminate or minimize the effect on the overall system performance. The classifications of FDI were divided into two major groups, which are modelbase and non model-based. Non model-based schemes are include Condition Monitoring and Intelligent FDI as shown in Fig.2

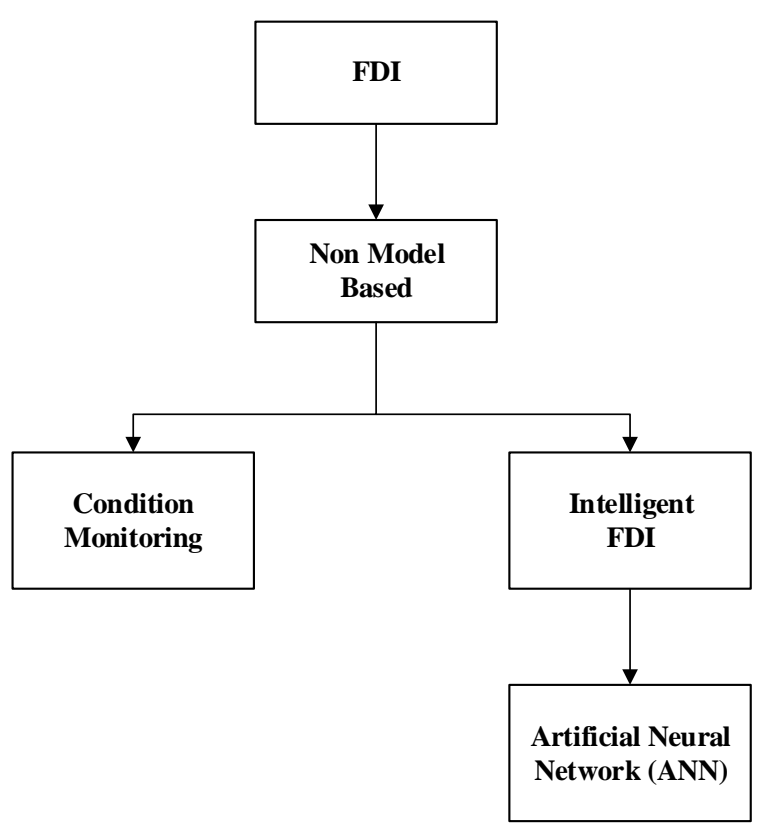

Fig.2 Fault Detection and Isolation (FDI)

\subsection{Artificial neural network}

The feed forward perceptron can be separated into Single Layer Perceptron (SLP) and Multilayer Perceptron (MLP). The multilayer perceptron consists of multiple neurons and multiple parallels. The multilayer perceptron include of the input layer, hidden layer and output layer is shown in Fig.3.

The output function can be expressed in term of the matrix as follow:

$$
y=f(W p+b)
$$


Where $p^{T}=\left[\begin{array}{llll}p_{1} & p_{2} & \ldots & p_{s}\end{array}\right]$ is the input of MLP $b^{T}=\left[\begin{array}{llll}b_{1} & b_{2} & \ldots & b_{R}\end{array}\right]$ is the bias of MLP and $W=\left(\begin{array}{ccc}W_{11} & \ldots & W_{1 R} \\ \vdots & \ddots & \vdots \\ W_{S 1} & \cdots & W_{S R}\end{array}\right)$ is weight of MLP

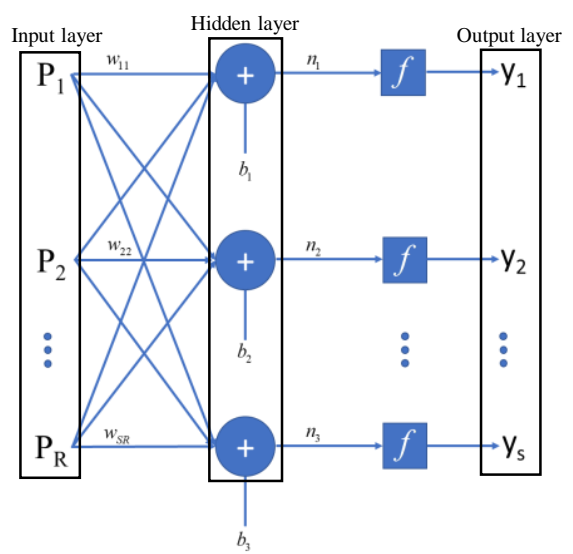

Fig.3 Multilayer Perceptron (MLP)

\subsection{Linear bearing model}

The linear bearing with recirculating ball bearings designed to use for move heavy loads along the precision linear path. The linear bearing consists of 2 major components with moving carriage and precision linear rail as shown in Fig.4. The ball or roller is used for the movement between carriage and linear rail with frictionless. According to continues run of linear bearing the contact between ball bearing and precision rail is the lead to worn out of mechanical part and degeneration of lubricant.

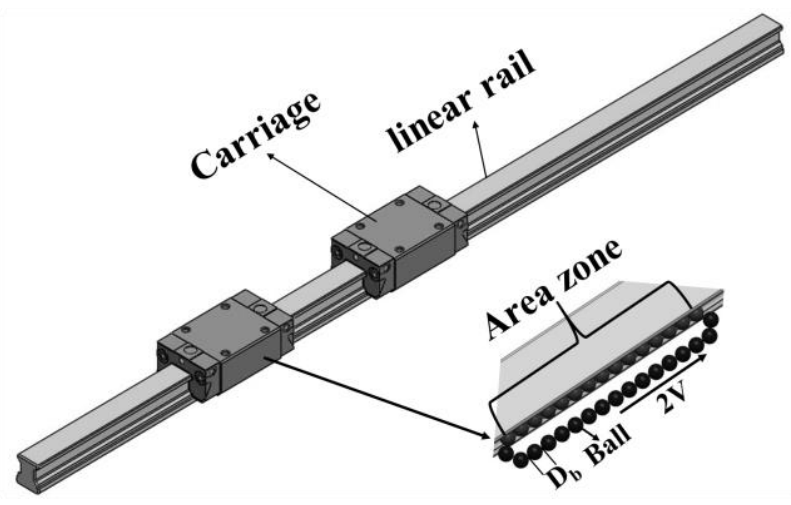

Fig. 4 Component and ball element of linear bearing.

To define the characteristic frequency of the ball bearing, the contact between ball bearing to rail and carriage each time and ball bearing completes rotation. The characteristic frequency of ball bearing is described as

$$
f_{\text {ball }}=\frac{2 V_{\text {ball }}}{\pi D_{b}}
$$

Where $V_{b a l l}$ is velocity of ball bearing and $D_{b}$ is diameter of ball bearing.

\subsection{Fast Fourier Transform (FFT) spectrum}

In general vibration signal was analyzed both time domain and frequency domain. The most common technique for fault diagnostic to identify the status of the machine is the vibration analysis. However, when the component of machine such as linear bearing moving it will be generated vibration signal at several frequencies. Therefor time domain analysis is difficult to extract the machine condition. Traditional analysis has generally relied upon spectrum analysis based on Fast Fourier Transform (FFT) spectrum which are the signal processing in frequency domain since was shown as eq.3. It is the most important technique to detect the fault frequency of fault linear bearing.

$$
X(f)=\frac{1}{T} \int_{-\frac{T}{2}}^{+\frac{T}{2}} x(t) \cdot e^{-i 2 \pi f t} d t
$$

\section{Experimental setup and procedure}

\subsection{Data collection}

In this research, the experimental setup was performed with using the linear DC Motor (SGL 100AUM3-PS4J model). The experiment was setup by installed a clamping unit with the load $7 \mathrm{~kg}$ on the top of linear DC motor. The acceleration sensor set up with the $X$-axis parallel to the direction of movement to the floor plane, $Y$-axis perpendicular to the $X$-axis and Z-axis perpendicular to the floor plan. Because the $X$-axis heavily affected by free movement but $Y$-axis and Z-axis fixed movement due to the mechanic of magnetic linear DC motor. Vibration Measurement by acceleration sensor and data acquisition via IOtech and EZ-Analyst software. The acceleration sensor measurement PCB Piezotronics (356A32 model) is employed in these experiments. The vibration sensitivity of sensor is $99.2 \mathrm{mV} / \mathrm{g}$ in $X$-axis direction, $98.1 \mathrm{mV} / \mathrm{g}$ in $Y$-axis direction and $101.1 \mathrm{mV} / \mathrm{g}$ in Z-axis direction respectively. Sampling frequency is 4000 $\mathrm{Hz}$. The operational condition of linear motor was setup based on current setting in production mode with acceleration $1.00 \mathrm{~m} / \mathrm{s}^{2}$, velocity of $0.5 \mathrm{~m} / \mathrm{s}$ and moving distance of $400 \mathrm{~mm}$ for data collection.

Table 1. Ball fault characteristic frequencies

\begin{tabular}{ccc}
\hline $\begin{array}{c}\text { Motor speed } \\
(\mathrm{m} / \mathrm{s})\end{array}$ & $\begin{array}{c}\text { Ball speed } \\
(\mathrm{m} / \mathrm{s})\end{array}$ & $\begin{array}{c}\text { Ball Frequency } \\
(\mathrm{Hz})\end{array}$ \\
\hline 0.50 & 0.25 & 53.00 \\
\hline
\end{tabular}




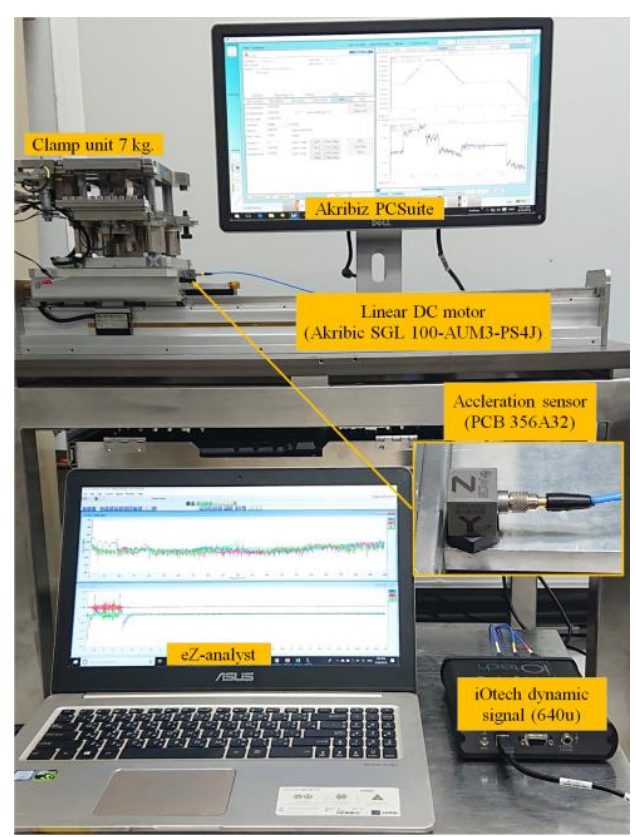

Fig.5 Experimental setup
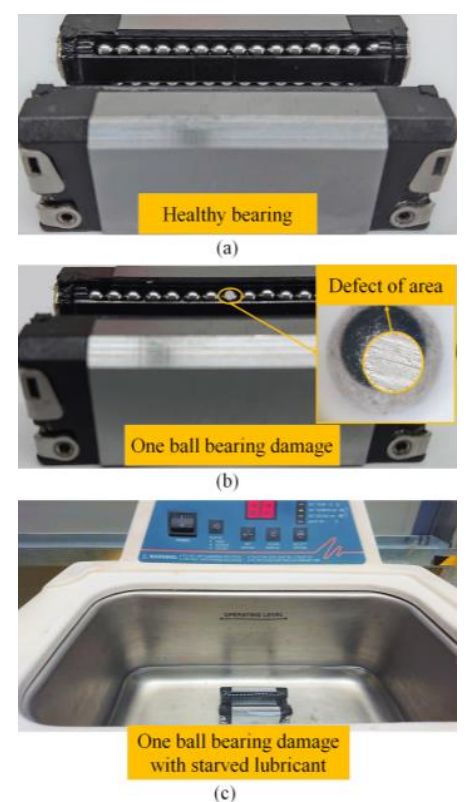

Fig. 6 (a) Healthy bearing (b) One ball bearing damage (c) One ball bearing damage with starved lubricant

The experimental setup is shown in Fig. 5. The data collection of three conditions were consisted of the healthy bearing, one ball bearing damage and one ball bearing damage with starved lubricant. The healthy bearing is perfect bearing is shown in Fig.6 (a).one ball bearing damage is simulate by manual gridding surface of ball bearing with area $2.565 \mathrm{~mm}^{2}$ as depicted in Fig. 6(b). The one ball bearing damage with starved lubricant was conducted by using isopropyl alcohol (IPA) cleaner and BRANSON (2510 model) digital ultrasonic cleaning to remove grease at carriage and rail as shown in Fig.6(c). Data collection by measuring vibration signal as above setting each condition are collect 50 data points total 150 data samples.

As setup motor speed $0.5 \mathrm{~m} / \mathrm{s}$ the characteristic frequency of ball bearing is calculate per equation (2) result shown as Table 1.

\subsection{Classification of Artificial Neural Network (ANN)}

The data of FFT spectrum is used for detecting and isolating of fault bearing conditions. These difficult to detection and isolation of fault bearing conditions in operational condition. In this section, detection and isolation of linear bearing condition require the ANN technique to forecasting models linear bearing condition. To simplified procedure the model, we selected 150 data samples to feed into the ANN models. Since the FFT spectrum values of various 3 bearing condition are very different.

Artificial neural network steps as 1 input layer, 1 hidden layer 10 nodes and 3 output as shown in Fig. 7. The ANN training was done the using the scaled conjugate gradient algorithm by dividing the data into $70 \%-15 \%-15 \%$ for training, validation and testing.

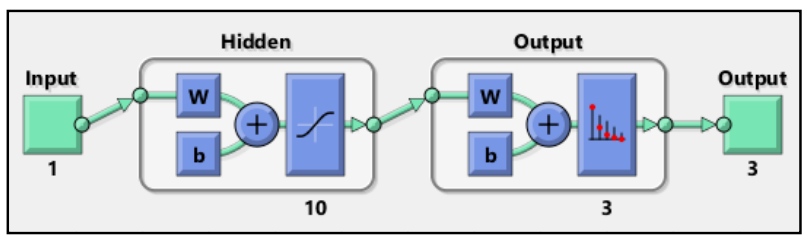

Fig.7 Artificial Neural Network (ANN)

\section{Result and discussion}

FFT spectrum data comparison for all 3 conditions considering that healthy bearing, one ball bearing damage and one ball bearing damage with starved lubricant were tested of linear bearing. Result shown as Fig. 8, Fig.9 and Table 2. Both fault bearing conditions are clearly segregate from healthy. Thus, in order to test the effectiveness of the proposed method for FFT spectrum. The FFT spectrum comparison of healthy bearing and fault bearing conditions. One ball bearing damage and one ball bearing damage with starved lubricant are increased by $18.52 \%$ and $11.62 \%$ respectively as shown in Table. 3 


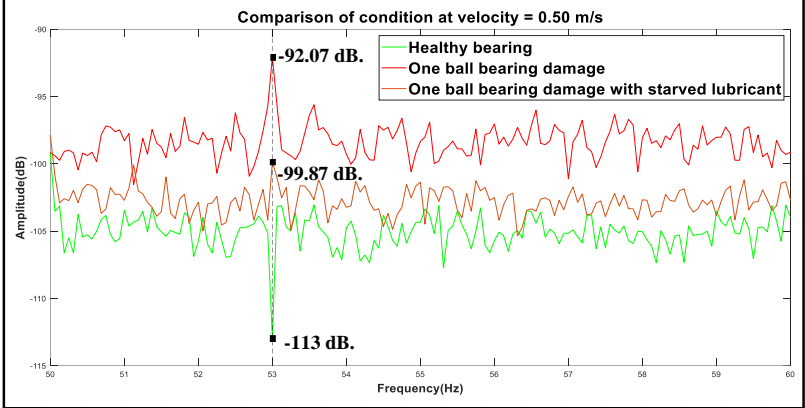

Fig.8 Frequency domain (dB) for velocity $0.50 \mathrm{~m} / \mathrm{s}$.

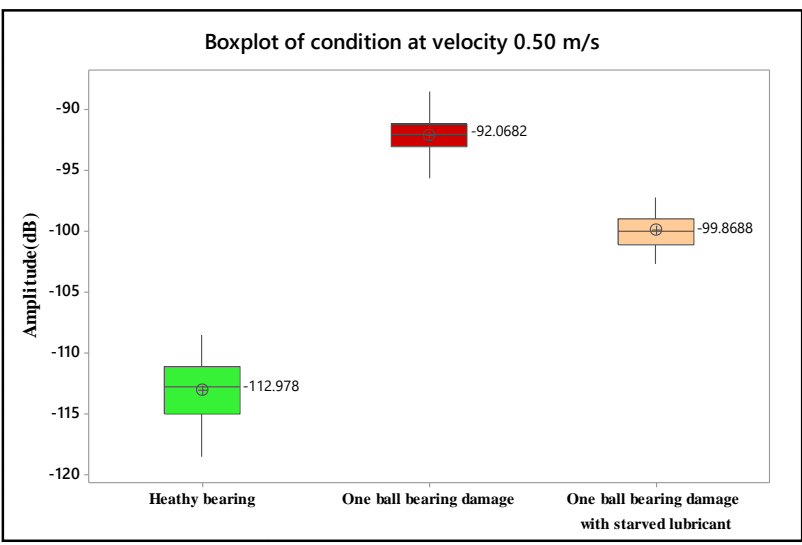

Fig.9 Boxplot comparison all 3 conditions at velocity $0.50 \mathrm{~m} / \mathrm{s}$

Table 2. Amplitude $(\mathrm{dB})$ at velocity $0.50 \mathrm{~m} / \mathrm{s}$

\begin{tabular}{lc}
\hline \multicolumn{1}{c}{ Condition } & $\begin{array}{c}95 \% \\
\text { Confidence interval }\end{array}$ \\
\hline Healthy bearing & -113.49 to 112.46 \\
One ball bearing damage & -92.58 to -91.55 \\
$\begin{array}{l}\text { One ball bearing damage } \\
\text { with starved lubricant }\end{array}$ & -100.38 to -99.35 \\
\hline
\end{tabular}

Table 3. Comparison of between heathy bearing and fault bearing conditions.

\begin{tabular}{cccc}
\hline Target & $\begin{array}{c}\text { Fault bearing } \\
\text { conditions }\end{array}$ & Delta & $\%$ \\
\hline Healthy bearing & One ball bearing damage & 20.93 & 18.52 \\
Healthy bearing & $\begin{array}{c}\text { One ball bearing damage } \\
\text { with starved lubricant }\end{array}$ & 13.13 & 11.62 \\
\hline
\end{tabular}

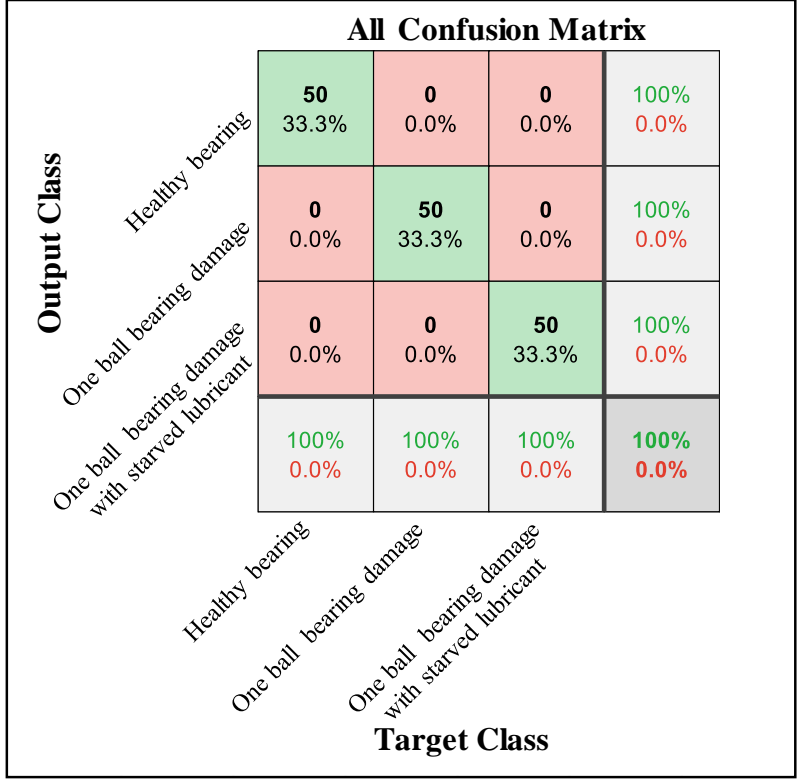

Fig.10 Confusion matrix

The ANN result shown the training stopped at $26^{\text {th }}$ iterations. The detection and isolation values of the linear bearing condition of ANN training. The ANN can satisfy effectiveness detect and isolate of the linear bearing condition. The confusion matrix is conducted by comparing the forecast accuracy convergence. The accuracy percentage of the accuracy is $100 \%$ as shown in Fig.10. The test results demonstrated the detection and isolation values with decision making of the ANN analysis.

\section{Conclusion}

FDI method can be used for reliability improvement of the automation machine. Premilary mechanical failure detection of the automation machine is very improtant in operational condition before the machine breaks down. This paper presents linear motor fault detection using FFT spectrum and ANN method in operational condition. The vibration amplitude can be analyzed the linear bearing faults at ball frequency of $53 \mathrm{~Hz}$, according to motor speed of $50 \mathrm{~m} / \mathrm{s}$. In case of healthy bearing has been compared with one ball bearing damage and one ball bearing damage with starved lubricant, the vibration amplitudes were increased $18.52 \%$ and $11.62 \%$ approximately. The decision making of ANN method can be correctly identified the linear bearing fault. The experimental results show potential application of ANN and FFT spectrum technique as FDI tool for linear bearing fault detection performance. 


\section{Acknowledge}

This research was supported by the Research and Researcher for Industry (RRI) under the Thailand Research Fund (TRF) offers a Scholarship for Ph.D. Students, Suranaree University of Technology (SUT) agency through grant PHD60I0043.

\section{References}

1. A.B. Jaouher, F. Nader, C.M. Brigitte, F. Farhat, Applied Acoustics, 89, 16-27(2015).

2. A. Issam, F. Nadir, B. Nadir, O. Brahim, D. Adel. Journal of Sound and Vibration, 397, 241-265(2017).

3. C. Jian, R.B. Robert, Mechanism and Machine Theory, 104, 161-176(2016).

4. L. Han, Z. Jianzhong, Z. Yang, J. Wei, Z. Yuncheng, ISA Transactions, 77, 167-178(2018).

5. C. Zhiqiang, D. Shengcai, C. Xudong, L. Chuan, S. Rene-Vinicio, Q. Huafeng, Microelectronics Reliability, 75, 327-333(2017).

6. S. Haidong, j. Hongkai, L. Xingqiu, L. Tianchen, Computer in Industry,96,27-39(2018)

7. Z. Jafar, A.T. Mohammad, R.K. Hamid, Mechatronics, 24,151-157(2014)

8. U. Muhammet, O. Mustafa, D. Mustafa, K. Haluk, Measurement,58,187-196(2014)

9. B. Cluaudio, I. Fabio, C. Marco, R. Riccardo, B. Alberto, IEEE Transactions on Industrial Electronics 58, 1684-1694(2011).

10. L. Chen, W. Zhenya, Z. Bo, Advanced Engineering Informatics, 32, 139-151(2017).

11. Z. Chuan, F. Zhipeng, W. Xiukun, Q. Yong. Expert Systems With Applications.108.233-245(2018)

12. A.Z.R Muhammad, A.N Shahab, A.B. Saeed, Neurocomputing, 219, 280-299(2017).

13. M. Bubathi, M.A. Sanjith, B. Kishnakumar, S.A.V. Satya Murty, Mechanical System and Signal Processing, 35, 150-166(2013). 